Die Lösungsmittel sind jeweils angegeben. Die Logarithmen von $\varepsilon$ stehen stets in Klammern hinter der Wellenlänge $\lambda_{\max }[\mathrm{m} \mu]$.

Herrn Dr. W. HüBeL, European Research Associates, Brüssel, und Frl. Dr. L. Vallarino, Mailand, danke ich für die Überlassung der Substanzen und Diskussionen recht herzlich. Herrn Prof. Dr. E. O. Fischer bin ich für die Förderung dieser Arbeit durch Überlassung der beiden von der Deutschen Forschung g e meins ch a f t, Bad Godesberg, zur Verfügung gestellten Spektralphotometer zu großem Dank verpflichtet. Herrn Dr. J. W. Miller bin ich für wertvolle Diskussionen über die UV-Spektren und Frl. H. HumMeL für ihre Hilfe bei der Messung der Spektren Dank schuldig.

\title{
Beziehungen der Infrarotschwingungen von 4-substituierten Benzonitrilen zur antibakteriellen Wirkung der aus ihnen synthetisierten Benzolcarbonsäurethioamide
}

\author{
Von Joachim Seydel \\ Aus dem Tuberkulose-Forschungsinstitut Borstel, Institut für experimentelle Biologie und Medizin \\ (Direktor: Prof. Dr. Dr. Enno Freerksen) \\ (Z. Naturforschg. 16 b, 419—422 [1961] ; eingegangen am 23. Februar 1961)
}

\begin{abstract}
Es werden IR-Spektren von $p$-substituierten Benzonitrilen diskutiert, insbesondere die Intensität der Nitril- und der Ringschwingung. Dabei ergibt sich eine Beziehung zur Ausbeute, zur Lage der $\mathrm{C}=\mathrm{S}$-Schwingung und zur minimalen in vitro-Hemmkonzentration der aus den Benzonitrilen synthetisierten Benzolcarbonsäurethioamide.
\end{abstract}

In Fortsetzung der Arbeit über die Zusammenhänge von Struktur und antibakterieller Wirkung gegenüber dem Mycobacterium tuberculosis und anderen Mykobakterien bei Benzolcarbonsäurethioamiden (Kaкimoto, Seydel und Wempe, 1961) ${ }^{1}$ wurden die ir-spektrographischen Untersuchungen auf die Synthesevorprodukte der Benzolcarbonsäurethioamide, die Nitrile, ausgedehnt.

Von der Beobachtung ausgehend, daß einige Nitrile bei der Umsetzung mit Schwefelwasserstoff in Gegenwart von Pyridinbasen mit hohen Ausbeuten das entsprechende Carbonsäurethioamid liefern, andere dagegen unter diesen Bedingungen schlecht oder gar nicht reagieren, wurden die IR-Spektren einiger Benzonitrile aufgenommen. Es fiel auf, daß Nitrile, die sich nicht so gut mit Schwefelwasserstoff umsetzen, Carbonsäurethioamide von größerer antibakterieller Wirksamkeit liefern.

Es schien uns daher von Nutzen zu sein, durch ir-spektrographische Untersuchungen Kenntnisse über die Elektronenverhältnisse an diesen Nitrilen, insbesondere an der Nitrilgruppe zu gewinnen, um daraus Schlüsse auf die Verhältnisse bei den entsprechenden Benzolcarbonsäurethioamiden ziehen zu können, bei denen infolge der schwierigen Zuord-

1 S. Kakimoto, J. Seydel u. E. Wempe, Jber. Borstel 5, im Druck. nung im Spektralbereich $1350-1250 \mathrm{~cm}^{-1}$ und der schlechten Löslichkeit die Aussagen unsicher blieben (Какімото und Mitarbb., 1961) ${ }^{1}$.

\section{Material und Methoden}

Die Untersuchungen wurden an den 10 Verbindungen der Tabelle durchgeführt. Diese Verbindungen wurden von uns synthetisiert und gereinigt. Die korrigierten Schmelzpunkte der Substanzen (Leitz Schmelzpunktmikroskop) stimmen mit den Literaturwerten hinreichend überein. Für die IR-Messungen wurde der vollautomatische Leitz-IR-Spektrograph mit linearer Wellenzahlskala verwendet (NaCl-Prisma, Meßbereich 4000 bis $650 \mathrm{~cm}^{-1}$, Spaltprogramm 3, Laufzeit 2,4 $\min / 500$ bzw. $100 \mathrm{~cm}^{-1}$ ). Die Messungen erfolgten:

a) an $\mathrm{KBr}$-Preßlingen, die $10 \mu$ Mole Substanz auf $1 \mathrm{~g} \mathrm{KBr}$ enthielten und bei $30 \mathrm{t}$ etwa 1 min lang gepreßt wurden,

b) in Chloroformlösung, die $100 \mu \mathrm{Mole} / \mathrm{ml}$ enthielt.

Es wurden Küvetten mit $\mathrm{NaCl}$-Fensterplatten von $45 \mathrm{~mm}$ Durchmesser und $5 \mathrm{~mm}$ Dicke mit einem $\mathrm{Ab}$ standsring von $0,1 \mathrm{~mm}$ verwendet. Die Wellenzahlskala wurde vor jeder Messung mit einer Polystyrolfolie $(0,05 \mathrm{~mm}$ dick $)$ auf die Banden bei 3060 und 3026 $\mathrm{cm}^{-1}$ geeicht. Von der Schwingung der $\mathrm{C} \equiv \mathrm{N}$-Gruppe ist die Maximal-Extinktion

$$
\varepsilon_{\max }=\frac{\ln I_{0} / I}{c l}
$$

angegeben. Zur Bestimmung der Intensität der Ringschwingung bei $\sim 1600 \mathrm{~cm}^{-1}$ wurde die Formel von 
RAMSAY $^{2}$ (1952) Methode I benutzt

$$
A=\frac{\pi}{2} \frac{1}{c l} \ln \left(\frac{I_{0}}{I}\right) v_{\max } \Delta v_{1 / 2} .
$$

Die IR-Daten für die Benzolcarbonsäurethioamide und ihre antibakterielle in vitro-Wirksamkeit sind der erwähnten Arbeit (Kакгмото u. Mitarbb. ${ }^{1}$, 1961, Tab. 5) entnommen.

\section{Ergebnisse und Diskussion}

Beim Vergleich der IR-Spektren einiger $p$-substituierter Benzonitrile zeigte sich, daß die Nitrilschwingung bei den verschiedenen Derivaten ziemlich lagekonstant ist. Bei sehr starkem + M-Effekt treten Verschiebungen bis maximal $25 \mathrm{~cm}^{-1}$ zu niedrigeren Wellenzahlen ein. Die Intensitäts-Differenzen sind dagegen beträchtlich. Die Auswertung der Messung

$$
\varepsilon_{\max }=\frac{\ln I_{0} / I}{c l}
$$

wird durch die ausgeprägte symmetrische Form der Nitrilbande und durch ihre isolierte Lage erleichtert. Thompson und Mitarbb. (1949) ${ }^{3}$. Kitson und Griffith $(1952)^{4}$. Lippert (1955) ${ }^{5}$. Sensi und Mitarbb. (1955) ${ }^{6}$, Skinner und Thompson $(1955)^{7}$ und Lippert und Vogel $(1956)^{8}$ haben bereits einige der hier angeführten Nitrile ir-spektrographisch untersucht und kommen, soweit Intensitätsmessungen vorliegen, zur gleichen Reihenfolge der Intensitätszunahme. Wir haben alle Substanzen noch einmal untersucht, um bessere Vergleichsmöglichkeiten zu haben.

Es ist elektronentheoretisch leicht erklärbar, daß die Extinktion mit zunehmendem + M-Effekt des $p$-Substituenten stark zunimmt, bei Substituenten mit - M-Effekt dagegen abnimmt (s. Tabelle). Die Intensität der Nitrilschwingung ist abhängig von der Polarisation und der Resonanzenergie der $\mathrm{C} \equiv \mathrm{N}$. Gruppe $(\mathrm{A}-\mathrm{B})$ und $(\mathrm{A}-\mathrm{C})$. Polarisation und

2 D. A. Ramsay, J. Amer. chem. Soc. 74, 72 [1952].

3 H. W. Thompson, R. R. Brattain, H. M. Randall u. R. S. Rasmussen, in: H. T. Clatke, I. R. Johnson and R. Robinson, The chemistry of penicillin. p. 382-414. Princeton Univ. Press. Princeton 1949.

4 R. E. Kitson u. N. E. Griffith, Analytic. Chem. 24, 334 [1952].

5 E. Lippert, Z. Elektrochem., Ber. Bunsenges. physik. Chem. 59, 534 [1955].

${ }^{6}$ P. Sensi u. G. G. Gallo, Gazz. chim. ital. 85, 235 [1955].

7 M. W. Skinner u. H. W. Thompson, J. chem. Soc. [London] 1955, 487.

8 E. Lippert u. W. Vogel, Z. physik. Chem. N.F. 9, 133 [1956].
Resonanzenergie wiederum sind abhängig von der Art der Substituenten.

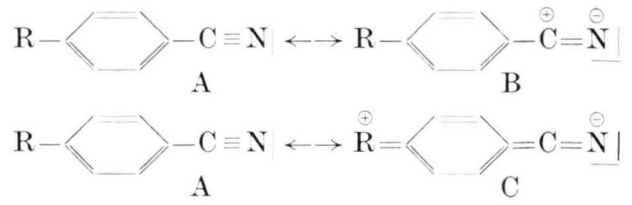

Die Vergrößerung von Dipolmoment und Resonanzenergie der Nitril-Gruppe wirkt sich ungünstig auf die Anlagerungs-Affinität von Schwefelwasserstoff aus, die Ausbeuten werden geringer * (KIndLeR, $1926^{9}$; TAYLoR, 1960) ${ }^{10}$, die antibakterielle in vitroWirkung der entsprechenden Carbonsäurethioamide dagegen wächst. Thompson und Steel (1956) ${ }^{11} \mathrm{er}$ wähnten bereits in einer Arbeit die Korrelation der Schwefelwasserstoff-Anlagerung zur Bandenintensität der Nitrilschwingung bei vier substituierten Benzonitrilen; ebenso geben sie für eine Reihe von Benzonitrilen die Beziehung der Nitril-Schwingungsintensität und Lage zu den $\mathrm{H}$ a m m e $\mathrm{t} t$ - Werten an. Die folgenden Abbildungen beschreiben die Bezie-

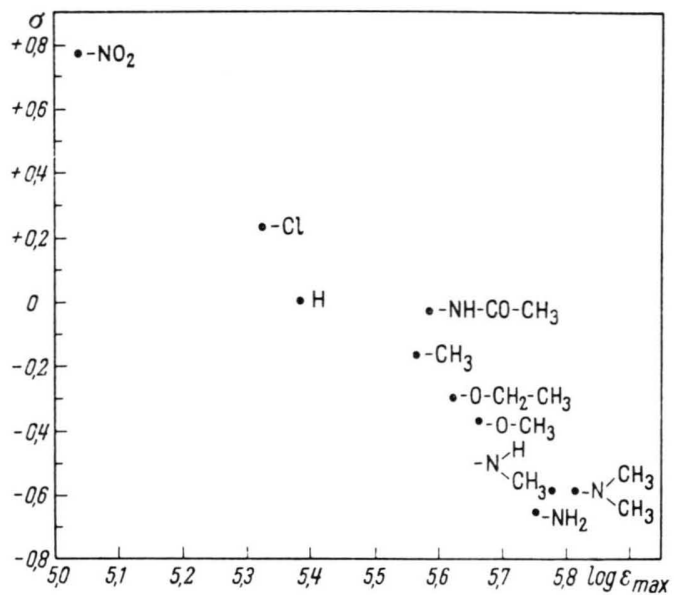

Abb. 1. Beziehung zwischen $\log \varepsilon_{\max }(\mathrm{C} \equiv \mathrm{N})_{y}$ und $\sigma$-Werten von substituierten Benzonitrilen in Chloroform.

* Setzt man die Ausbeute bei der Umsetzung der Nitrile zu den Carbonsäurethioamiden mit Schwefelwasserstoff bei Benzonitril $=100$, so ergibt sich für $p$-Chlor- 493, $p$-Methyl- 51,9 und für $p$-Methoxybenzonitril 4l,6 (persönliche Mitteilung von SEKIKAWA $\left.{ }^{12}, 1960\right)$.

9 K. Kindler, Liebigs Ann. Chem. 450, 1 [1926].

10 E. C. Taylor u. J. A. Zoltewicz, J. Amer. chem. Soc. 82, 2656 [1960].

11 H. W. Thompson u. G. Steel, Trans. Faraday Soc. 52, 1451 [1956].

12 I. Sekikawa, Res. Inst. Tuberc. Hokkaido Univ., Sapporo, Japan, pers. Mitt. (1960). 


\begin{tabular}{|c|c|c|c|c|c|c|c|c|c|c|c|c|}
\hline & \multicolumn{5}{|c|}{$\mathrm{R}=\mathrm{C} \equiv \mathrm{N}$} & \multicolumn{7}{|c|}{$\mathrm{R}=\mathrm{C}_{\backslash \mathrm{NH}_{2}}^{/ / \mathrm{S}}$} \\
\hline & \multirow[b]{2}{*}{ 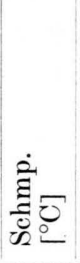 } & \multirow[b]{2}{*}{ 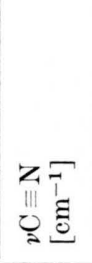 } & \multirow{2}{*}{ 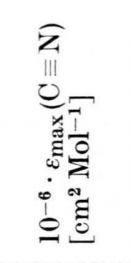 } & \multirow[b]{2}{*}{ 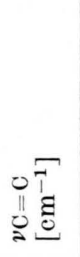 } & \multirow{2}{*}{$\begin{array}{l}0 \\
\| \\
0 \\
0 \\
4 \\
0 \\
0 \\
0 \\
0 \\
0\end{array}$} & \multirow[b]{2}{*}{ 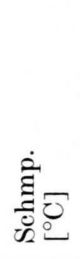 } & \multirow[b]{2}{*}{ 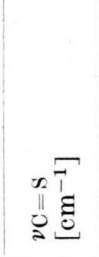 } & \multirow{2}{*}{ 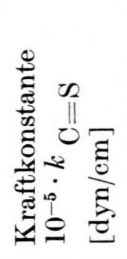 } & \multicolumn{4}{|c|}{$\begin{array}{l}\text { Minimale Hemm- } \\
\text { konzentration }(\mu \mathrm{Mol} / 1\end{array}$} \\
\hline & & & & & & & & & 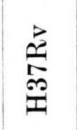 & 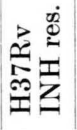 & ల్ల & 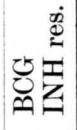 \\
\hline & 148 & $\begin{array}{c}2238 \\
(\mathbf{2 2 3 6})\end{array}$ & $\begin{array}{c}0,1114 \\
(\mathbf{0 , 3 6 1 6})\end{array}$ & 1605 & 5,69 & $\begin{array}{l}175 \\
\text { bis } \\
178\end{array}$ & ? & $?$ & 1024 & 1024 & 512 & 512 \\
\hline & 91 & $\begin{array}{c}2232 \\
(\mathbf{2 2 2 9})\end{array}$ & $\begin{array}{c}0,2112 \\
(\mathbf{0 , 1 7 8 0})\end{array}$ & 1602 & 3,45 & 134 & 1322 ? & 8,99 & 512 & 512 & 512 & 512 \\
\hline & $\begin{array}{l}\text { Sdp. } \\
187\end{array}$ & 2230 & 0,2415 & 1603 & 1,15 & 123 & 1335 ? & 9,17 & 2048 & 2048 & 2048 & 2048 \\
\hline & 28 & 2230 & 0,3683 & 1608 & 3,47 & 172 & 1325 & 9,035 & 512 & 512 & 512 & .512 \\
\hline $\mathrm{H}_{3} \mathrm{C}-\mathrm{C}$ & 208 & 2230 & 0,3795 & 1609 & $?$ & 233 & $?$ & $?$ & 512 & 512 & 256 & 256 \\
\hline $\mathrm{H}_{3} \mathrm{C}-\mathrm{CH}_{2}-$ & 59 & 2227 & 0,4201 & 1609 & 10,88 & 162 & 1315 & 8,90 & 512 & 128 & 128 & 128 \\
\hline & 66 & 2225 & 0,4515 & 1609 & 11,92 & 154 & 1289 & 8,55 & 64 & 32 & 64 & 32 \\
\hline & 86 & $\begin{array}{l}2219 \\
(\mathbf{2 2 1 5})\end{array}$ & $\begin{array}{c}0,5656 \\
(\mathbf{0 , 6 1 9 0 )}\end{array}$ & 1609 & 14,10 & 182 & 1282 & 8,46 & 64 & 64 & 64 & 64 \\
\hline $\mathrm{H}_{3} \mathrm{C}^{\prime}$ & 60 & $\begin{array}{l}2219 \\
(\mathbf{2 2 1 5})\end{array}$ & $\begin{array}{c}0,6038 \\
(\mathbf{0 , 6 1 4 4})\end{array}$ & 1615 & 22,57 & 212 & - & - & 64 & 32 & 64 & 64 \\
\hline $\mathrm{H}_{3} \mathrm{C}^{N}$ & 75 & $\begin{array}{c}2219 \\
(\mathbf{2 2 1 5})\end{array}$ & $\begin{array}{c}0,6483 \\
(\mathbf{0 , 6 3 1 9})\end{array}$ & 1611 & 42,13 & 256 & 1285 & 8,50 & 64 & 32 & 32 & 32 \\
\hline
\end{tabular}

Halbfetter Druck: KBr-Preßling; gewöhnlicher Druck: in Chloroformlösung.

Tab. 1. Infrarotdaten von $p$-substituierten Benzonitrilen und den entsprechenden Carbonsäurethioamiden und deren minimale in vitro-Hemmkonzentrationen gegen einige Mykobakterien-Stämme.

hung für die eigenen Meßwerte. Es werden die $\sigma$-Werte nach $\mathrm{J}$ a f f é benutzt und gegen die von uns ermittelten $\varepsilon_{\max }$-Werte der $\mathrm{C} \equiv \mathrm{N}$-Schwingung bzw. gegen deren Wellenzahlen aufgetragen. Man erhält eine lineare Abhängigkeit über einen weiten Bereich der $\sigma$-Werte.

Darüber hinaus besitzen die $p$-substituierten Benzolringe eine Ringschwingung zwischen 1640 bis $1545 \mathrm{~cm}^{-1}$ und eine weitere bei $\sim 1500 \mathrm{~cm}^{-1}$. Das Intensitätsverhältnis dieser Banden ändert sich ebenfalls mit dem Wechsel des zur Nitrilgruppe $p$-ständigen Substituenten. Im Falle der $p$-substituierten Benzonitrile wächst die Intensität der Ringschwingung bei $\sim 1605 \mathrm{~cm}^{-1}$ (s. auch Lippert, $1955)^{5}$ infolge starker Konjugation, die die chinoide Struktur begünstigt. Die Intensität dieser Ringschwingung ist bei $p$-Dimethylaminobenzonitril, das

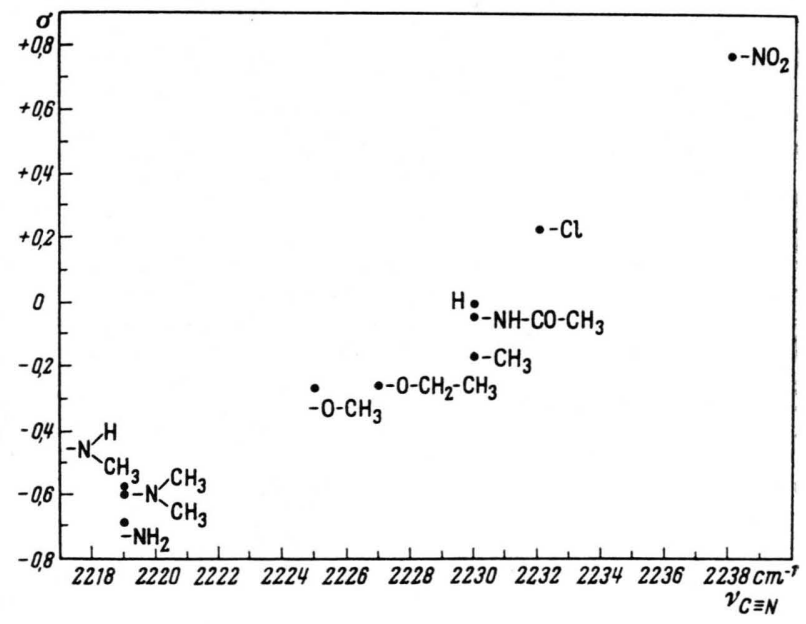

Abb. 2. Beziehung zwischen $v_{(\mathrm{C} \equiv \mathrm{N})}$ und $\sigma$-Werten von substituierten Benzonitrilen in Chloroform. 
von den hier untersuchten $p$-substituierten Nitrilen den stärksten + M-Effekt besitzt, am größten und zeigt, daß die Moleküle ähnlich der in der Grenzform $\mathrm{C}$ beschriebenen Ladungsverteilung vorliegen.

Die in der vorangegangenen Arbeit (Какімото und Mitarbb., 1961) ${ }^{1}$ versuchte Zuordnung der Lage der $\mathrm{C}=\mathrm{S}$-Schwingungen (bzw. der daraus berechneten Kraftkonstanten) zur antibakteriellen in vitroWirkung dieser Präparate war in einigen Fällen wegen der Schwierigkeit der Identifizierung der $\mathrm{C}=\mathrm{S}$-Schwingung nicht ganz eindeutig. Trotzdem glaubten wir aus den vorliegenden Befunden schließen zu können, daß mit zunehmender Polarisation $\operatorname{der} \mathrm{C}=\mathrm{S}$-Bindung die antibakterielle Wirksamkeit der Carbonsäurethioamide steigt.

Bei den Ausgangsverbindungen dieser Carbonsäurethioamide, den Nitrilen, liegen die Verhältnisse günstiger. Die bessere Löslichkeit der Nitrile macht Intensitätsmessungen möglich. Aus der Elektronenverteilung an der $\mathrm{C} \equiv \mathrm{N}$-Gruppe des dem jeweiligen Carbonsäurethioamid zugehörigen Nitrils kann man eine Bestätigung der in der Arbeit von Какімото und Mitarbb. (1961) ${ }^{1}$ vertretenen Auffassung über Struktur und Wirkung bei Benzolcarbonsäurethioamiden ableiten. Die Beeinflussung der Elektronenkonfiguration der $-\mathrm{C} \equiv \mathrm{N}$-, bzw. der $-\mathrm{CS}-\mathrm{NH}_{2}$ Gruppe, die beide Substituenten mit - M-Effekt sind, durch die gleichen $p$-Substituenten sollte gleichsinnig erfolgen, ebenso die der Ringschwingung, so daß man von den Verhältnissen bei den Nitrilen auf die bei den Carbonsäurethioamiden schließen darf.

Die Elektronenverteilung an der $\mathrm{C}=\mathrm{S}$-Gruppierung und ihre dadurch bedingte Reaktionsfähigkeit ist mit großer Wahrscheinlichkeit verantwortlich für die antibakterielle Wirksamkeit der Benzolcarbonsäurethioamide; der $p$-Substituent modifiziert diese Wirksamkeit durch Beeinflussung der Elektronenverteilung an der $\mathrm{C} \equiv \mathrm{N}$ - bzw. $\mathrm{C}=\mathrm{S}$-Bindung, die polarisiert sein muß.

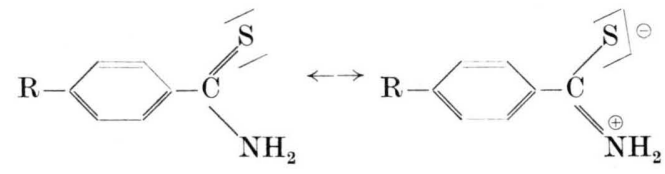

Möglicherweise wird die polarisierte $\mathrm{C}=\mathrm{S}$-Bindung durch Resonanz mit einer polaren bzw. chinoiden Struktur und Konjugation mit dem aromatischen Ring begünstigt und stabilisiert. Danach erwarten -wir, daß die Umsetzung von $p$-substituierten Benzonitrilen, die eine starke Intensität der Nitrilund Ringschwingung haben, zu Carbonsäurethioamiden mit größerer antibakterieller in vitro-Wirkung führt. Die bisherigen Befunde haben diese Erwartung bestätigt.

\title{
Die demische Natur der „Luftphytoncide“ von Blattpflanzen insbesondere von Robinia pseudacacia
}

\author{
II. Mitteilung über Abwehrstoffe von Pflanzen² \\ Von H. Schildknecht * und G. Rauch ** \\ Aus dem Institut für Organische Chemie der Universität Erlangen \\ (Z. Naturforschg. 16 b, 422-429 [1961] ; eingegangen am 21. März 1961)
}

\begin{abstract}
Mit einer Versuchsanordnung, bei der nur gasförmige, flüchtige Stoffe erfaßt werden, wurde das Phytoncid von Robinia pseudacacia aus dem Pflanzenbrei isoliert und durch Zonenschmelzen und normales Erstarren angereichert. Durch sein 2.4-Dinitrophenylhydrazon, sein UV- und IR-Absorptionsspektrum sowie durch Synthese konnte es als das $\Delta^{2}$-Hexenal identifiziert werden. Pharmakologisch wirkt es wenig toxisch, zeigt aber als Phytoncid fungizide und protistozide Eigenschaften. Es wurde weiterhin als Phytoncid der Eiche, Erle, Lupine, der schwarzen Johannisbeere, der Heidel- und Preißelbeere sowie des Ligusterstrauches und des Grases erkannt. In Freilandversuchen konnte nachgewiesen werden, daß $\Delta^{2}$-Hexenal auch von unverletzten Pflanzen ausgeschieden wird.
\end{abstract}

Bereits vor rund 30 Jahren hat Tokis ${ }^{1}$ den Begriff Phytoncide geprägt; er bezeichnete so alle Stoffe mit protistozider, bakterieller und fungizider Wirkung.

\footnotetext{
* Teilweise vorgetragen auf dem IV. Internationalen Kongreß für Biochemie in Wien 1958.
}

die besonders von höheren Pflanzen ausgeschieden werden. Inzwischen aber versteht der russische Forscher unter Phytoncide einen ganzen Stoffkomplex

\footnotetext{
1 Siehe hierzu B. P. Tокіn, Phytoncide, VEB Verlag Volk und Gesundheit, Berlin 1956.
} 